\title{
Adaptation of the diaphragm and the vastus lateralis in mild-to-moderate COPD
}

\author{
M. Doucet*, R. Debigaré*, D.R. Joanisse*, C. Côté ${ }^{\text {, }}$ P. LeBlanc*, J. Grégoire*, J. Deslauriers*, \\ R. Vaillancourt*, F. Maltais*
}

Adaptation of the diaphragm and the vastus lateralis in mild-to-moderate COPD. M. Doucet, R. Debigaré, D.R. Joanisse, C. Côté, P. LeBlanc, J. Grégoire, J. Deslauriers, R. Vaillancourt, F. Maltais. C) ERS Journals Ltd 2004.

ABSTRACT: The chronology of diaphragm and vastus lateralis adaptation in chronic obstructive pulmonary disease (COPD) has not been studied. The hypothesis of this study was that muscle changes would occur earlier in the diaphragm than in the vastus lateralis in COPD, a finding that would suggest that local factors would be more important than systemic factors in determining the muscle phenotypic expression, at least in mild-to-moderate disease.

The adaptation of the vastus lateralis and diaphragm muscles was evaluated in patients with mild-to-moderate COPD and in subjects with normal pulmonary function.

In both groups, the oxidative potential and the number of lipofuscin inclusions were higher in the diaphragm than in the vastus lateralis. Compared to control, the diaphragm in COPD had a higher oxidative capacity and a higher proportion of type I fibres, with a reciprocal decrease in type IIA fibres, while there was no difference in diaphragmatic cross sectional areas, capillarisation and lipofuscin inclusions. No significant differences were found in the vastus lateralis from both groups.

In conclusion, these data indicate that the diaphragm in controls and in chronic obstructive pulmonary disease has a higher oxidative potential than the vastus lateralis, and that muscle adaptation occurs earlier in the diaphragm than in the vastus lateralis in mild-to-moderate chronic obstructive pulmonary disease.

Eur Respir J 2004; 24: 971-979.
*Centre de Recherche de 1'Hôpital Laval, Institut Universitaire de Cardiologie et de Pneumologie de 1'Université Laval, "Division de Kinésiologie, Université Laval, and "Centre de Recherche du CHUQ Pavillon CHUL, Université Laval, Québec, Canada.

Correspondence: F. Maltais, Centre de Pneumologie, 2725 Chemin Ste-Foy, Ste-Foy, Québec, G1V 4G5, Canada

Fax: 14186564762

E-mail: medfma@hermes.ulaval.ca

Keywords: Adaptation

chronic obstructive pulmonary disease

diaphragm

vastus lateralis

Received: April 162004

Accepted after revision: July 302004

This study was supported by Canadian Institutes of Health Research grant MOP-53135. $\mathrm{R}$. Debigaré was the recipient of a $\mathrm{PhD}$ training award from the Fonds de la Recherche en Santé du Québec. F. Maltais is a research scholar of the Fonds de la Recherche en Santé du Québec.
Skeletal muscles have a great capacity to adapt to a variety of chronic perturbations. In severe chronic obstructive pulmonary disease (COPD), evidence of peripheral muscle adaptation includes a shift of type I towards type II fibres [1], a decrease in muscle capillarisation of all fibre types [2] and reduced oxidative enzyme activities [3]. Not surprisingly, adaptation of the diaphragm has also been described in severe COPD. This muscle is chronically challenged by the increase in respiratory load and by hyperinflation, which places the diaphragm in a mechanically disadvantageous position, constituting a chronic training stimulus over time [4]. Evidence of diaphragmatic adaptation has been mostly reported in patients with advanced COPD, in whom a shift from type II to type I fibres has been found, along with an increase in mitochondrial density [5-7]. The general conclusion arising from these studies is that the vastus lateralis muscle and the diaphragm adapt in a different and opposite manner in severe COPD.

One limitation of the current literature is that the diaphragm and the vastus lateralis muscle samples were not obtained from the same individuals. This raises methodological concerns, given the large interindividual variability in fibre-type distribution and enzymatic activities [8]. For instance, in one large study, the proportion of type I fibres in the vastus lateralis in sedentary healthy subjects varied $15-85 \%$ [8]. In this context, a difference in the proportion of type I fibres between the vastus lateralis and the diaphragm obtained from different groups of patients may be very difficult to interpret. In order to address this issue and to rule out the potential confounding effects of differences in sex, nutritional status and previous exposure to corticosteroids, vastus lateralis and diaphragm samples were obtained from the same individuals in this study. Recently, ENGELEN et al. [9] reported a higher type I fibre proportion in the diaphragm than in the vastus lateralis obtained from the same individuals with COPD. However, muscle capillarisation and enzymatic activities, two other important indices of oxidative capacity, were not evaluated in this study.

Although important changes are noted in the diaphragm and the vastus lateralis in COPD, the chronology of these adaptations has not been studied; for instance it is unknown whether diaphragmatic adaptation precedes or follows the peripheral muscle adaptation. The answer to this question would help elucidating the underlying mechanisms of skeletal muscle dysfunction in COPD. The ideal experimental design to address this question would be a longitudinal study evaluating how the diaphragm and the vastus lateralis adapt over time in a given individual during the progression of 
his/her COPD; however, this would be, for obvious reasons, an extremely challenging study to do. The current authors reasoned that some interesting information about the chronology of muscle adaptation in COPD could be obtained by studying a group of patients with mild-to-moderate disease. If skeletal muscle adaptation in COPD is initiated by a systemic process, such as low-grade chronic inflammation, one would expect a similar and concurrent adaptation in the diaphragm and the vastus lateralis muscle. Conversely, the working hypothesis was that muscle changes would occur earlier in the diaphragm than in the vastus lateralis of patients with COPD, a finding that would be consistent with the fact that local factors (e.g. the degree of muscle activation) would be more important than systemic factors (e.g. systemic inflammation) in determining the muscle phenotypic expression, at least early in mild-to-moderate disease.

The present study was, therefore, undertaken 1) to compare histochemical, morphological and enzymatical changes in the vastus lateralis and diaphragm muscles within the same individuals, both in patients with mild-to-moderate COPD and in subjects with normal lung function; and 2) to evaluate whether evidence of muscle adaptation occurs earlier in the diaphragm than in the vastus lateralis muscle in mild-tomoderate COPD.

\section{Methods}

\section{Subjects}

Twenty-eight subjects undergoing lung resection were recruited. Sixteen subjects had normal lung function (13 males/three females) and twelve patients had COPD (10 males/two females). The diagnosis of COPD was based on a previous smoking history and pulmonary function testing showing irreversible airflow obstruction (post-bronchodilator forced expiratory volume in one second (FEV1) $<80 \%$ predicted and FEV1/forced vital capacity $<70 \%$ ). Patients with comorbid conditions that could influence skeletal muscle function were excluded: heart failure, uncontrolled diabetes, systemic corticosteroids, low body weight (body mass index (BMI) $<20 \mathrm{~kg} \cdot \mathrm{m}^{-2}$ ) and unstable nutritional status. Nutritional status was evaluated with anthropometric parameters, as well as with albumin level, at the time of the investigation for lung cancer. Body composition was not assessed in this study. The institution's Ethics Committee approved the study protocol and each patient signed a written informed consent form.

\section{Evaluation}

Pulmonary function tests and anthropometric measurements. Standard pulmonary function tests, including spirometry, lung volumes using body plethysmography and diffusion capacity, were obtained according to previously described guidelines [10], and related to normal values of KNUDSON et al. [11], and GOLDMAN and BECKLAKE [12], and Cotes and HALL [13], respectively. Height and weight were measured in both groups according to standardised methods [14].

Muscle biopsies. Diaphragm biopsy. Diaphragm biopsy was obtained during the thoracic surgical procedure. Either the right or left diaphragm was biopsied, depending on the side of the surgery. The surgeon was careful in ensuring that muscle samples came from the same area of the costal median region of the diaphragm (control group, right side $=10$ out of 16 ; COPD, right side=nine out of 12). After the diaphragmatic fascia was visualised and cut, a section of the costal median diaphragm was carefully dissected along its longitudinal axis to obtain specimens weighing $\approx 250-500 \mathrm{mg}$. Diaphragm specimens were frozen into liquid nitrogen and stored at $-70^{\circ} \mathrm{C}$ for future analysis.

Quadriceps biopsy. Needle biopsies of the vastus lateralis, as described by BERGSTRÖM [15] and routinely done in the current authors' laboratory [2], were performed within $24-48 \mathrm{~h}$ after the diaphragm biopsy. Muscle specimens were immediately frozen using the method described above for the diaphragm samples.

\section{Skeletal muscle and data analysis}

For each subject, three adjacent muscle sections were selected, and used to determine fibre typing, capillarisation and lipofuscin content (fig. 1). All techniques have been previously described in detail [2, 16, 17] and were done without knowledge of the clinical data or from which muscle the samples were coming from.

Fibre typing and cross-sectional area (CSA) determination. One muscle section was histochemically stained for myofibrillar ATPase activity, according to the single-step ethanol-modified technique [18]. Muscle fibres were classified according to the staining intensity: type I (nonstained), type IIA (lightly stained) and type IIB (darkly stained). All muscle sections were magnified and transferred to
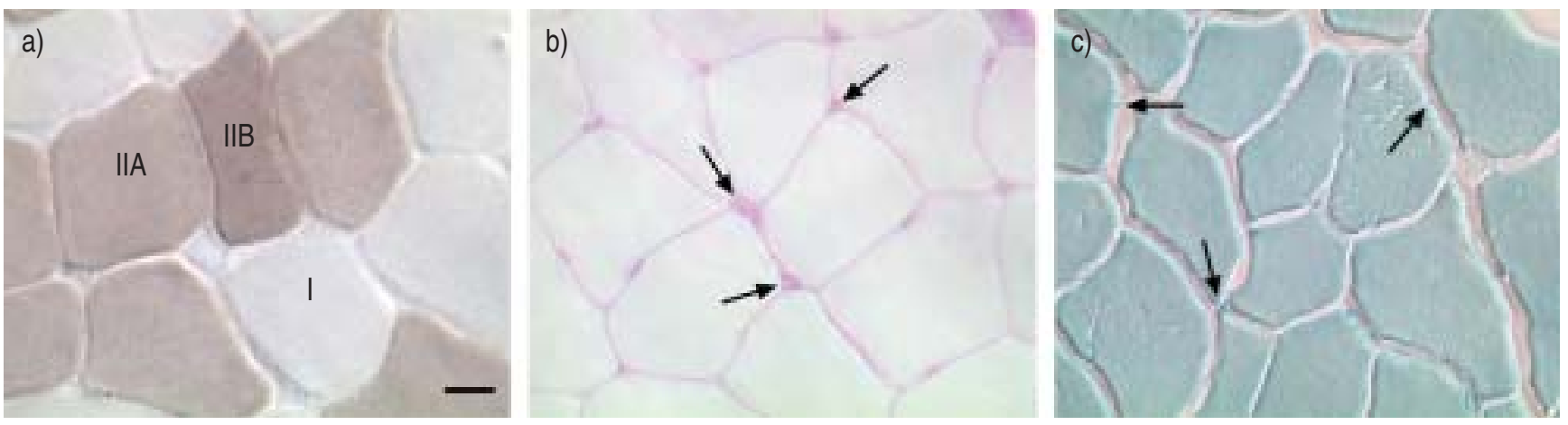

Fig. 1.-a) Histochemical staining (myofibrillar ATPase activity) for fibre typing and cross-sectional areas. Fibre types are indicated on the figure. b) $\alpha$-Amylase-periodic acid shift staining for muscle capillarisation (arrows). c) Ferric-ferricyanide reduction staining for lipofuscin determination (arrows). Internal scale bar $=20 \mu \mathrm{m}$. 
an image analysing system (Image Pro Plus 4.1 for Windows; Media Cybernetics, Silver Spring, MD, USA). All fibres were counted and classified to obtain the fibre-type composition for each subject. The cross-sectional areas (CSA) of each fibre-type was calculated based on 40 randomly selected fibres of each type [19]. Histochemistry was preferred over immunohistochemistry, because of the difficulty in obtaining reliable antibodies to subclassify type II fibres in humans. In a validation study, it was found that both methods $[18,20]$ were highly concordant; the proportion of type II fibres obtained from each method was strongly correlated (slope $=0.96, r=0.83, p<0.001$ ).

Muscle capillarisation. One muscle section was stained with the $\alpha$-amylase-periodic acid shift method to visualise capillarisation [2]. Two indexes of muscle capillarisation were used: 1) the capillary-to-fibre ratio, obtained by dividing the total number of capillaries by the total number of fibres on corresponding muscle sections; and 2) the capillary contacts/ fibre CSA ratio, obtained by dividing the number of capillary contacts by the mean CSA of each fibre type. This was done on 20 fibres randomly selected for each type [2].

Muscle lipofuscin. Lipofuscin is a marker of accumulative oxidative stress. After routine fixation in paraformaldehyde $4 \%$ in phosphate buffer solution ( $\mathrm{pH} 7.3$ ), muscle sections were stained with the ferric-ferricyanide reduction test technique [17]. The ratio of lipofuscin inclusions/fibre was obtained by dividing the total number of inclusions by the total number of fibres. The methodology related to this measurement can be found in greater detail elsewhere [17].

Enzyme activity determination. The activities of two oxidative enzymes, citrate synthase (CS) and 3-hydroxyacyl CoA dehydrogenase (HADH), and three glycolytic enzymes, lactate dehydrogenase (LDH), phosphofructokinase (PFK) and hexokinase (HK), were measured using standard spectrophotometric techniques, as described previously [16]. Each assay was done in duplicate and the average of the two values is reported. The characteristics of the reaction media used for each enzyme have been previously reported [16].

\section{Statistical analysis}

Results are expressed as mean $\pm \mathrm{SD}$, according to the normality of the data. Subjects' characteristics and the lung function indices for both groups were compared using unpaired t-tests or Mann-Whitney U-tests. Muscle enzymatic activities were compared using the enzymatic ratios of glycolysis to citric acid cycle and to the $\beta$-oxidation of the fatty acids. These discriminating ratios provide useful indications about the preferential enzymatic pathways in the muscle [21]. A crossed-nested analysis of variance was applied to compare the diaphragm and the vastus lateralis within and between individuals. To proceed with the analysis, a model was used with the compound-symmetry structure, which has constant variance and constant covariance between measurements. The normality assumption was verified with the ShapiroWilk test. The Brown and Forsythe's variation of Levene's test statistic was used to verify the homogeneity of variances. Results were considered significant with p-values $<0.05$.

\section{Results}

\section{Subjects' characteristics}

Subjects' characteristics and pulmonary function data are provided in table 1. Patients with COPD had, on average,
Table 1.-Patient characteristics

\begin{tabular}{lcc}
\hline & Control & COPD \\
\hline Subjects & 16 & 12 \\
Sex M:F & $13: 3$ & $10: 2$ \\
Age yrs & $58 \pm 11$ & $65 \pm 8$ \\
BMI kg·m ${ }^{-2}$ & $28 \pm 4$ & $26 \pm 4$ \\
FEV1 L & $2.8 \pm 0.5$ & $1.7 \pm 0.4^{* * *}$ \\
FEV1\% pred & $95 \pm 13$ & $65 \pm 8^{* * *}$ \\
FEV1/FVC \% & $75 \pm 7$ & $54 \pm 9^{* * *}$ \\
FRC \% pred & $102 \pm 33$ & $131 \pm 27^{*}$ \\
RV\% pred & $112 \pm 35$ & $150 \pm 37^{*}$ \\
RV/TLC \% & $37 \pm 8$ & $47 \pm 10^{* *}$ \\
DL,CO \% pred & $103 \pm 19$ & $71 \pm 31^{* *}$ \\
Albumin g. L ${ }^{-1}$ & $36 \pm 6$ & $39 \pm 5$ \\
Length of hospitalisation days & $10^{\#}$ & $7^{\#}$ \\
Pathological lung cancer staging & & \\
Ia & 1 & 2 \\
Ib & 4 & 2 \\
IIa & 1 & 2 \\
IIb & 3 & 1 \\
IIIa & 1 & \\
IIIb & 1 & \\
IV & 1 & \\
Others & & \\
Bronchogenic cyst & 1 & 1 \\
Bullectomy & & \\
Intrabronchial harmartoma & 1 & \\
Granuloma & & \\
Benign pulmonary nodule & 1 & \\
Pulmonary infarct & & \\
Unique metastasis & 1 & \\
\hline
\end{tabular}

COPD: chronic obstructive pulmonary diseasec; M: male; F: female; BMI: body mass index; FEV1: forced expiratory volume in one second; FVC: forced vital capacity; FRC: forced residual capacity; RV: residual volume; TLC: total lung capacity; $D$ L,CO: diffusing capacity. Data are presented as $\mathrm{n}$ or mean \pm SD. ${ }^{\#}$ : median value. ${ }^{* * *}: \mathrm{p}<0.001 ; * *: \mathrm{p}<0.01$; $*: \mathrm{p}<0.05$.

mild-to-moderate airflow obstruction, with a mean FEV1 of $65 \pm 8 \%$ pred. Based on the 2003 Canadian COPD guidelines [22], nine out of 12 patients had mild COPD (FEV1 of $60-75 \%$ pred), while the remaining three patients with COPD had moderate disease (FEV1 50-60\% pred). Airflow obstruction was associated with gas trapping and hyperinflation. BMI and albumin levels were similar between the two groups. According to the preoperative evaluation, 21 out of 28 subjects were thought to have a resectable lung cancer, while the remaining subjects had a benign tumour $(\mathrm{n}=6)$ or a unique pulmonary metastasis $(n=1)$. Five patients were found to have an unresectable lung cancer at the time of the surgery: malignant pleural effusion $\left(\mathrm{T}_{4}\right.$ tumour, $\left.\mathrm{n}=2\right)$, ipsilateral nodal metastasis in patient with negative mediastinoscopy $\left(\mathrm{N}_{2}\right.$ nodes, $\left.\mathrm{n}=2\right)$ and intrapulmonary metastasis $(n=1)$. These patients were equally distributed between both groups (COPD group $n=3$; control group $n=2$ ) and were not different preoperatively in terms of their BMI and clinical status than those who had complete surgical resection. Only one patient belonging to the COPD group received two cycles of neo-adjuvant chemotherapy for a $\mathrm{N}_{2}$ disease diagnosed pre-operatively. Clinical data from this patient were similar to the rest of the COPD group and removing her data from the analysis did not change the results. It was, therefore, decided to keep this subject in the study.

Comparison of the diaphragm and the vastus lateralis in the control group

Histochemical and morphological characteristics. The mean total number of fibres counted was similar in both muscles: 
$634 \pm 321$ (range 217-1263) for the diaphragm and $657 \pm 463$ (210-1777) for the vastus lateralis. The fibre-type distribution and CSA of all fibre-types were similar for both muscles (fig. 2a and b). The capillary-to-fibre ratio was also similar in the diaphragm and the vastus lateralis: $1.73 \pm 0.59$ versus $1.67 \pm 0.47$, respectively. However, the diaphragmatic type I fibres tended to have a better capillary contacts/fibre CSA ratio compared to the vastus lateralis (fig. 2c).

Muscle enzyme activity. Group mean values for muscle enzymatic activities are shown in table 2. Oxidative enzyme (CS, HADH) activities were markedly higher in the diaphragm compared to the vastus lateralis in control subjects. There was no significant difference in LDH activity between diaphragm and vastus lateralis. However, the PFK activity was lower and the HK activity was higher in the diaphragm compared to the
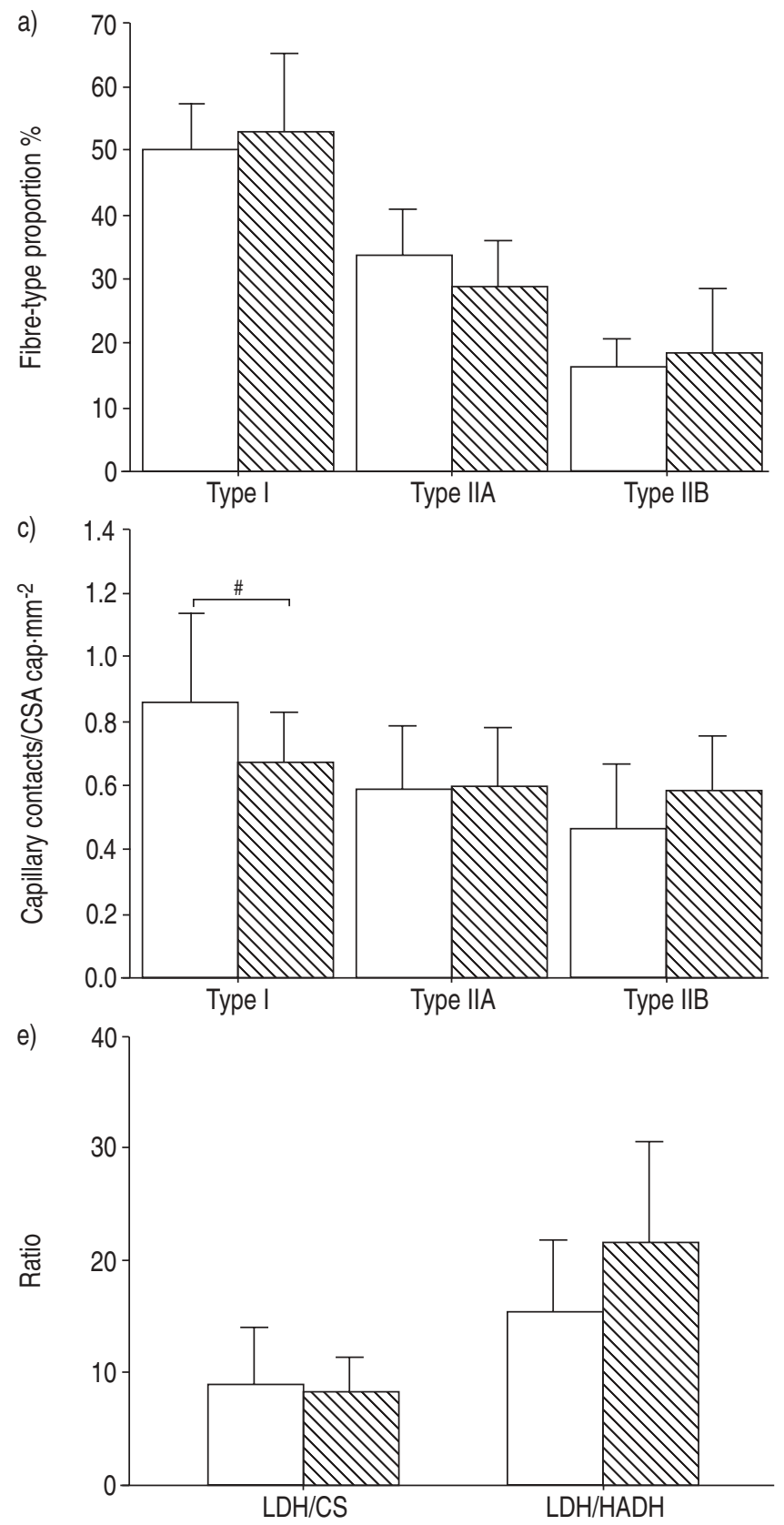

vastus lateralis. The discriminating enzyme ratios obtained in the diaphragm muscle and the vastus lateralis are provided in figure $2 \mathrm{~d}$ and e. The smaller ratios (PFK/CS, PFK/HADH) observed in the diaphragm indicate a higher potential for aerobic than for anaerobic energy metabolism [21] than in the vastus lateralis. Lastly, the number of lipofuscin inclusions per fibre was significantly higher in the diaphragm as compared to the vastus lateralis $(1.81 \pm 0.90$ versus $1.47 \pm 0.99, \mathrm{p}<0.05)$, an observation in agreement with their documented high oxidative potential.

Comparison of the diaphragm and the vastus lateralis in patients with COPD

Histochemical and morphological characteristics. The mean total number of fibres counted was similar in both muscles:
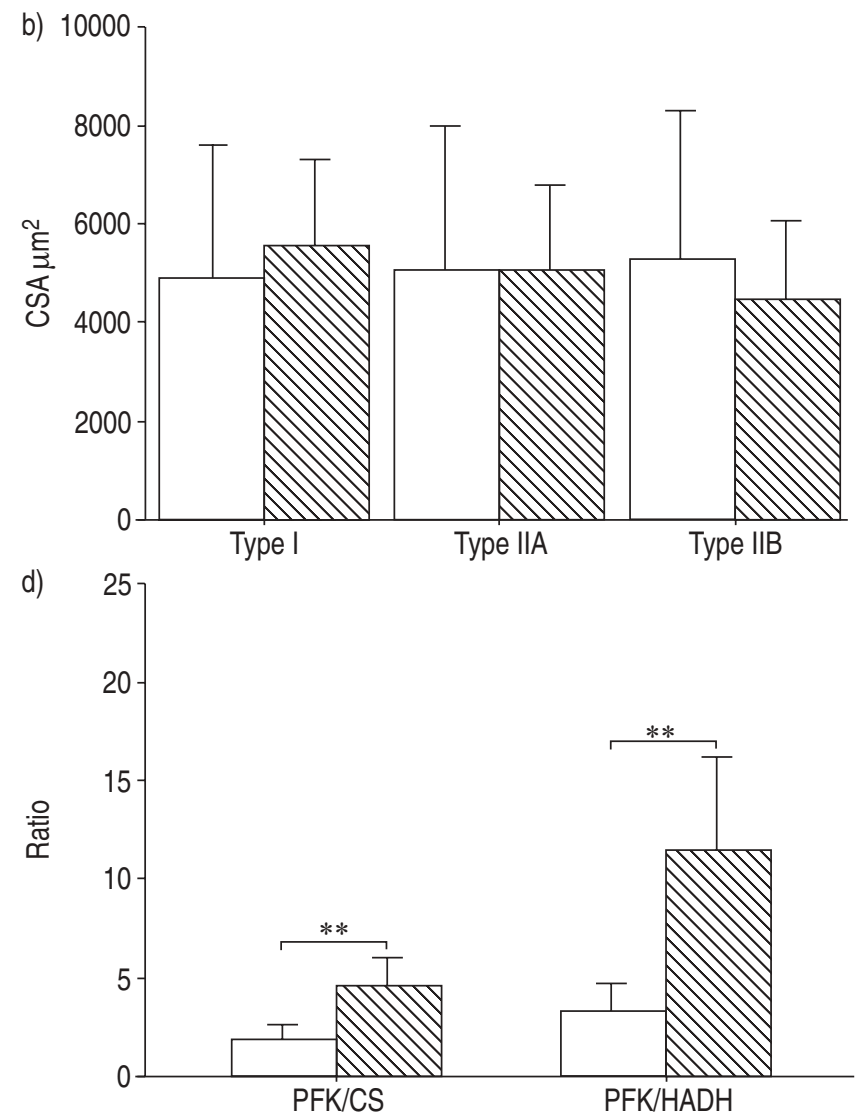

Fig. 2. - Comparison of the diaphragm ( $\square$ ) with the vastus lateralis $(\mathbb{\nabla})$ in the control group. a) Both muscles had similar fibre-type proportion and b) fibre cross-sectional areas (CSA). c) Capillary contacts/fibre CSA in type I tended to be higher in the diaphragm compared to the vastus lateralis. A significantly greater oxidative capacity was found in the diaphragm compared to the vastus lateralis, as indicated by d) a reduction in phosphofructokinase (PFK)/citrate synthase (CS) and PFK/3-hydroxyacyl CoA dehydrogenase (HADH) ratios in the former muscle, e) while the lactate dehydrogenase $(\mathrm{LDH}) / \mathrm{CS}$ and LDH/HADH ratios were similar between the two muscles. Data are presented as mean \pm SD. ${ }^{*}: \mathrm{p}=0.051 ;{ }^{* *}: \mathrm{p}<0.01$. 


\begin{tabular}{lcccc}
\hline & \multicolumn{2}{c}{ Control } & & COPD \\
\cline { 2 - 3 } & Diaphragm & Vastus lateralis & Diaphragm & Vastus lateralis \\
\hline Oxidative enzymes & & & & \\
CS & $18.02 \pm 5.11$ & $13.94 \pm 2.62^{* *}$ & $21.75 \pm 5.13$ & $12.98 \pm 3.02^{*}$ \\
HADH & $9.93 \pm 2.42$ & $5.98 \pm 2.35^{* * *}$ & $10.75 \pm 2.00$ & $4.91 \pm 1.00^{* * *}$ \\
Glycolytic enzymes & & & $105.92 \pm 33.35^{*}$ & $122.49 \pm 38.98$ \\
LDH & $143.92 \pm 53.32$ & $113.72 \pm 36.07$ & $31.06 \pm 10.31$ & $58.67 \pm 21.49^{*}$ \\
PFK & $30.34 \pm 8.16$ & $62.21 \pm 16.00^{* * *}$ & $1.37 \pm 0.48$ & $1.02 \pm 0.26^{*}$ \\
HK & $1.39 \pm 0.60$ & $0.89 \pm 0.56^{* * *}$ & & \\
\hline
\end{tabular}

Data are presented as $\mu \mathrm{mol} \cdot \mathrm{min}^{-1} \cdot \mathrm{g}^{-1}$ of muscle. COPD: chronic obstructive pulmonary disease; CS: citrate synthase; HADH: 3-hydroxyacyl CoA dehydrogenase; LDH: lactate dehydrogenase; PFK: phosphofructokinase; HK: hexokinase. *: $\mathrm{p}<0.05$; **: $\mathrm{p}<0.01$; ***: $\mathrm{p}<0.001$ versus the diaphragm in the corresponding group; ${ }^{\#}: \mathrm{p}<0.05$ versus the diaphragm in controls. Control $\mathrm{n}=16$, COPD $\mathrm{n}=12$.

$442 \pm 258(161-904)$ in the diaphragm and 626 $\pm 241(186-1,000)$ in the vastus lateralis. There was a significantly higher proportion of type I fibres in the diaphragm compared to the vastus lateralis, with no significant differences in the proportions of type IIA and type IIB fibres between the two muscle groups (fig. 3a). Fibre CSA (fig. 3b) and the capillaryto-fibre ratio $(1.69 \pm 0.29$ versus $1.59 \pm 0.30)$ were similar in the diaphragm and the vastus lateralis. As was the case in control subjects, diaphragmatic type I fibres tended to have a better capillary contacts/fibre CSA compared to the vastus lateralis (fig. 3c).

Muscle enzyme activity. The oxidative enzyme activities were markedly higher in the diaphragm compared to the vastus lateralis (table 2). PFK activity was much lower in the diaphragm than in the vastus lateralis muscle, while the opposite was observed for HK activity. All discriminating enzymatic ratios indicated a greater oxidative potential in the diaphragm compared to the vastus lateralis (fig. $3 \mathrm{~d}$ and e). Again, the number of lipofuscin inclusions per fibre was significantly higher in the diaphragm compared to the vastus lateralis: $2.23 \pm 0.66$ versus $1.57 \pm 0.65, \mathrm{p}<0.05$.

\section{Comparison of diaphragms between control subjects and patients with COPD}

Histochemical and morphological characteristics. The histochemical and morphological characteristics of the diaphragm in both groups of patients are presented in figure 4. Compared to control subjects, the diaphragm in COPD had a greater proportion of type I fibres with a reciprocal decrease in the proportion of type IIA fibres. Fibre CSA did not differ significantly between the two groups. The capillary-to-fibre ratios in control subjects $(1.73 \pm 0.59)$ versus COPD patients $(1.69 \pm 0.29)$, and the capillary contacts/fibre CSA in all fibre-type, were similar in both groups.

Muscle enzyme activity. Except for LDH, whose activity was reduced in COPD compared to control subjects, the enzymatic activities were comparable between the diaphragms in both groups. However, when considering discriminating enzymatic activity ratios, the diaphragm in COPD patients demonstrated an enhanced oxidative potential compared to the diaphragm in control subjects (fig. 4e). There was no statistically significant difference in lipofuscin accumulation between the diaphragm in COPD patients and in control subjects: $2.23 \pm 0.66$ versus $1.81 \pm 0.90$.

\section{Comparison of the vastus lateralis between controls and patients with COPD patients}

No significant difference in histochemical, morphological characteristics, muscle enzymatic activities or accumulation of lipofuscin was found when comparing the vastus lateralis of controls and patients with COPD.

\section{Discussion}

Previous studies have demonstrated that the diaphragm and the vastus lateralis undergo important adaptations in severe COPD $[6,23,24]$. The current study provides a detailed morphometric and metabolic comparison of these two muscles within the same individuals with COPD or normal pulmonary function. This allows controlling for potential confounding variables, such as the age, nutritional status and use of corticosteroids, in the comparison between the two muscles. Moreover, mild-to-moderate COPD patients were studied to allow some inference about the chronology of muscle adaptation in COPD. The main findings of the present study are consistent with previous publications, showing that the diaphragm is a more oxidative muscle compared to the vastus lateralis. In mild-to-moderate COPD, evidence of muscle adaptation was found in the diaphragm and not in the vastus lateralis. This differential adaptation is consistent with the hypothesis that, at a milder stage of the disease, local factors (e.g. the level of muscle activation) are probably more important than systemic factors to explain COPD-induced muscle adaptation.

\section{Muscle enzyme activities and morphological characteristics}

The present data in subjects with normal pulmonary function is consistent with numerous reports showing that the diaphragm is more oxidative $[25,26]$ as compared to the vastus lateralis. This trend is even more pronounced in patients with COPD. Indeed, oxidative enzyme activities were upregulated in this group (CS and HADH), which led to lower discriminating enzymatic ratios in the diaphragm when compared to the vastus lateralis. This suggests that the diaphragm in COPD greatly relies on its oxidative machinery, with a lesser dependence on anaerobic glycolysis. The tendency for a better diaphragmatic capillary contacts/fibre CSA as compared with the vastus lateralis is also in line with this observation.

The diaphragmatic adaptation of patients with advanced COPD was recently evaluated [5, 6, 23]. Compared to control 

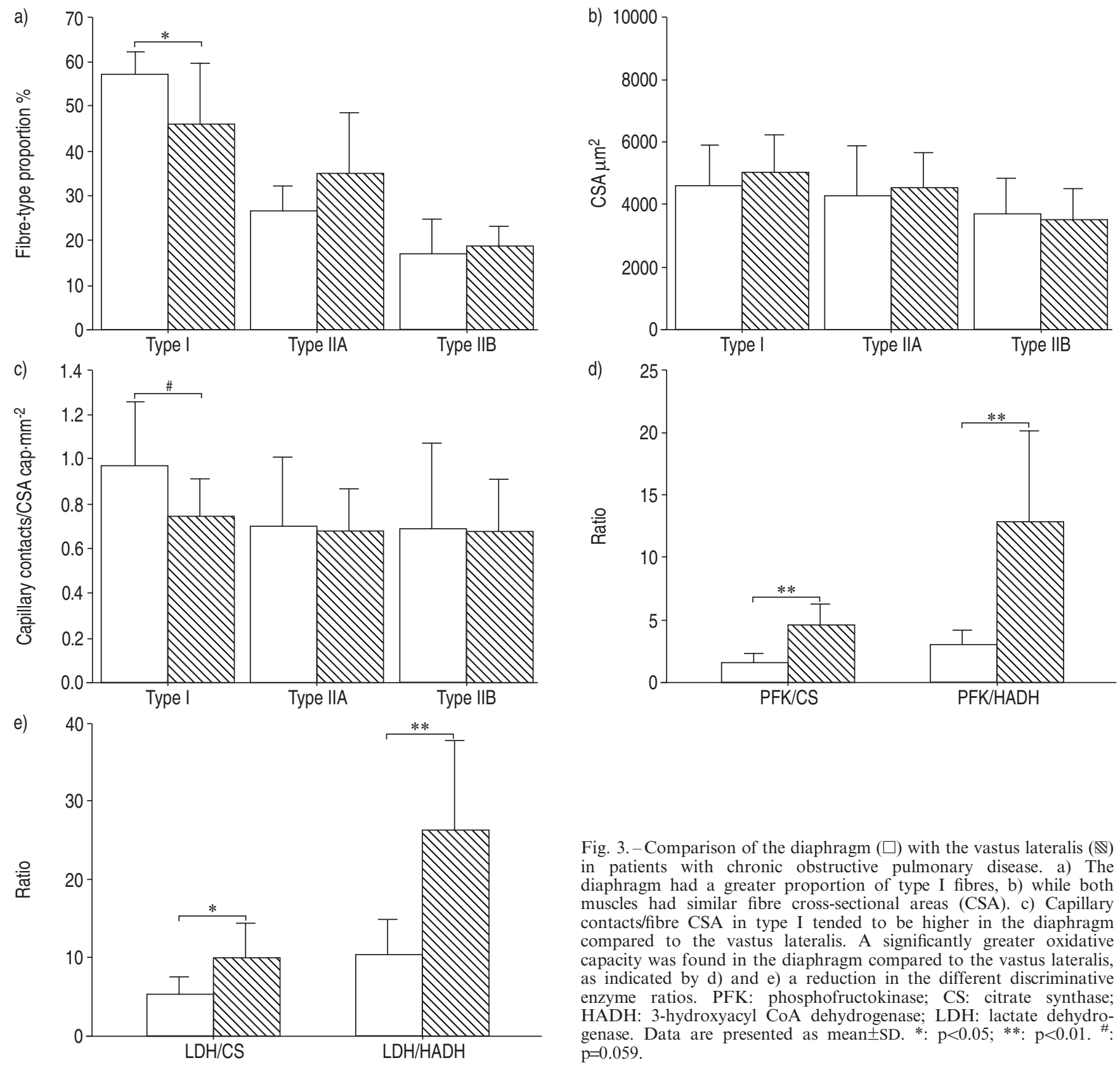

Fig. 3. - Comparison of the diaphragm $(\square)$ with the vastus lateralis ( $\mathbb{\mathbb { N }}$ ) in patients with chronic obstructive pulmonary disease. a) The diaphragm had a greater proportion of type I fibres, b) while both muscles had similar fibre cross-sectional areas (CSA). c) Capillary contacts/fibre CSA in type I tended to be higher in the diaphragm compared to the vastus lateralis. A significantly greater oxidative capacity was found in the diaphragm compared to the vastus lateralis, as indicated by d) and e) a reduction in the different discriminative enzyme ratios. PFK: phosphofructokinase; CS: citrate synthase; HADH: 3-hydroxyacyl CoA dehydrogenase; LDH: lactate dehydrogenase. Data are presented as mean \pm SD. *: $\mathrm{p}<0.05 ; * *: \mathrm{p}<0.01 .{ }^{\#}$ : $\mathrm{p}=0.059$.

diaphragms, a clear fibre-type shift from type II to type I fibres, a parallel reduction in the proportion of fast relative to slow myosin heavy chain, and increased mitochondrial capacity and efficiency [7] were found in the diaphragm of patients with advanced COPD [5, 23]. The present study is one of the few evaluating morphological and enzymatic activity of the diaphragm in mild-to-moderate COPD [27]. While previous investigators failed to report modification in the fibre-type profile in mild COPD, the current results demonstrate a shift in the same direction in terms of fibre type proportions, but of smaller magnitude than in patients with severe COPD $[5,6,23]$. The decrease in discriminating enzyme ratios (LDH/CS and LDH/HADH) in the diaphragm of patients with COPD as compared to controls is consistent with the fibre-type switch toward more oxidative fibres in the former individuals. Possible explanations for the discrepancy between these results and previous studies may lie within the

characteristics and size of the patient's population selected. Altogether, the adaptation found in the diaphragm of patients with COPD appears similar to what would be expected from an endurance training programme [28,29], except for the fact that no evidence of diaphragmatic adaptation in terms of muscle capillarisation was found in COPD. This is somewhat surprising considering the link between increased oxidative potential and the capillary supply [26]. In severe COPD, the diaphragm adapts by increasing both its mitochondrial and capillary density [30], suggesting that capillary proliferation is a late process in the adaptation.

In contrast to what was found at the diaphragmatic level, the vastus lateralis of patients with mild-to-moderate COPD patients did not differ from that of controls. The situation in mild COPD, therefore, differs from what is seen in severe COPD, where profound changes in the vastus lateralis muscle consistent with a reduced oxidative potential have been 

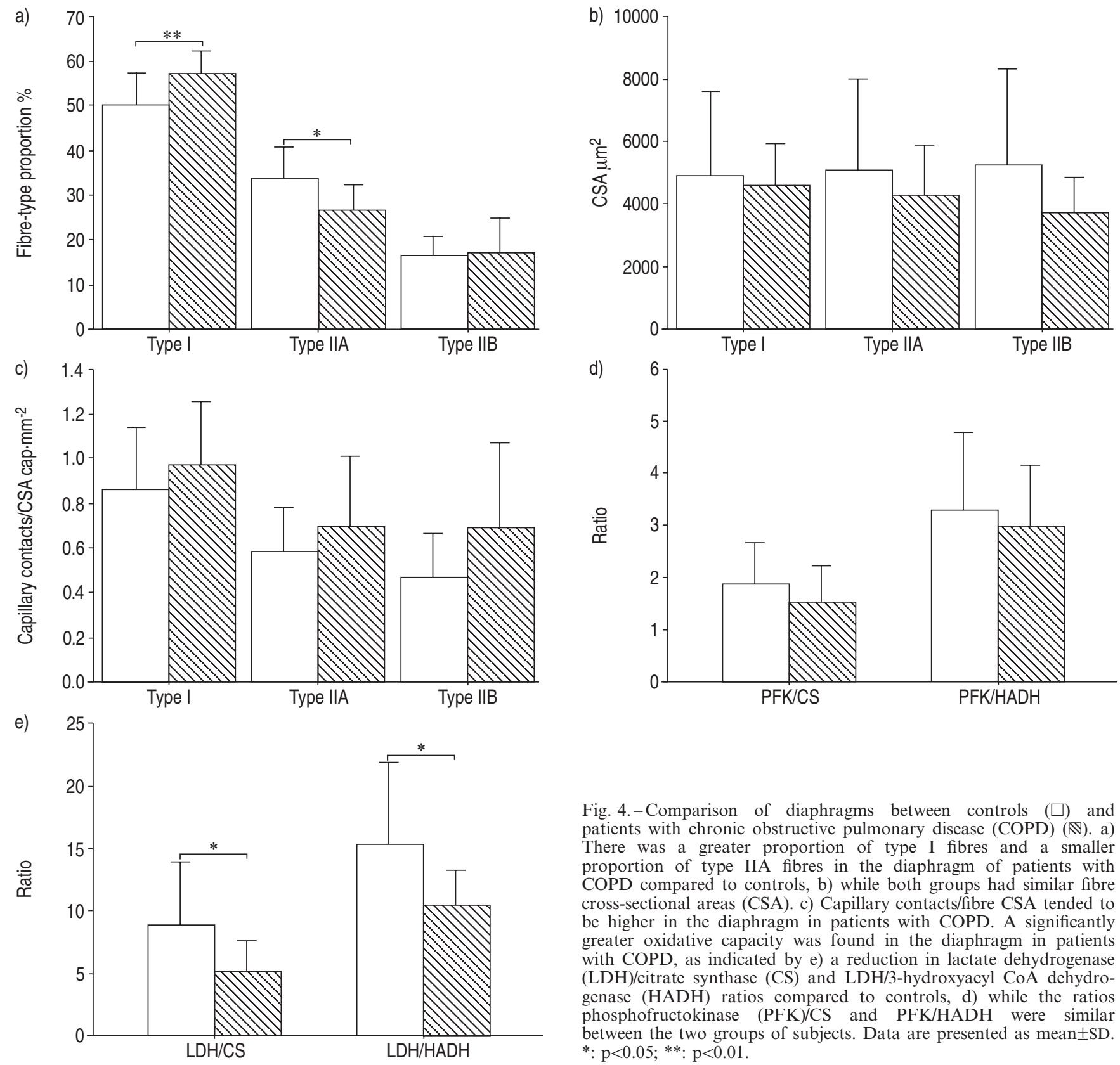

Fig. 4. - Comparison of diaphragms between controls ( $\square$ ) and patients with chronic obstructive pulmonary disease (COPD) $(\mathbb{Q})$. a) There was a greater proportion of type I fibres and a smaller proportion of type IIA fibres in the diaphragm of patients with COPD compared to controls, b) while both groups had similar fibre cross-sectional areas (CSA). c) Capillary contacts/fibre CSA tended to be higher in the diaphragm in patients with COPD. A significantly greater oxidative capacity was found in the diaphragm in patients with COPD, as indicated by e) a reduction in lactate dehydrogenase (LDH)/citrate synthase (CS) and LDH/3-hydroxyacyl CoA dehydrogenase (HADH) ratios compared to controls, d) while the ratios phosphofructokinase (PFK)/CS and PFK/HADH were similar between the two groups of subjects. Data are presented as mean \pm SD. $*: \mathrm{p}<0.05 ; * *: \mathrm{p}<0.01$.

repeatedly reported $[1,16,24]$. Overall, the data collected on both the diaphragm and vastus lateralis muscles strongly suggest that COPD-induced cellular adaptation occurs first in the diaphragm and later on in the vastus lateralis.

It is currently suggested that the diaphragm in stable COPD is a well adapted muscle, perhaps because is it protected by a state of chronic activation. The consistent finding of diaphragmatic fibre atrophy in COPD $[5,6,31]$ is, therefore, unexpected, because exercise training is usually associated with fibre hypertrophy. In the current study, fibre CSA tended to be smaller in the diaphragm compared to the vastus lateralis in both controls subjects and patients. Interestingly, muscle fibre atrophy has been reported after years of pure endurance training [32]. The significance and the mechanisms of this observation, which may represent an attempt to increase the number of capillaries per fibre CSA and reduce diffusion distance, may only be speculated upon.

\section{Markers of oxidative stress}

The choice of lipofuscin as a marker of oxidative stress was based on several observations. Although lipofuscin is a mixture of different substances, lipid peroxidation, a wellrecognised consequence of oxidative stress, is essential to its formation $[33,34]$. Lipofuscin accumulates when the load of free radicals is increased [35], and there is an inverse correlation between the level of tissue antioxidants and lipofuscin [36, 37]. A unique characteristic of lipofuscin is its poor elimination by cells [34]. As such, it was used as a marker of cumulative oxidative damages within the muscle fibres. The intracellular location of lipofuscin in the subsarcolemmal area (fig. 1c), a region rich in mitochondria, an important source of reactive oxygen species generation, is also consistent with the relationship between oxidative stress and lipofuscin. One methodological consideration when 
quantifying oxidative stress by counting the number of lipofuscin inclusions is that their surface area could vary from one inclusion to the next, so that a greater lipofuscin accumulation (the product of the number of inclusions by their surface) could vary despite a similar number of inclusions. To address this issue, the surface of all lipofuscin inclusions was measured in 40 randomly selected diaphragmatic and vastus lateralis fibres in five controls and five patients with COPD. Although there was some variation in size, the average surface area of the lipofuscin inclusions did not differ significantly between the two muscle groups.

Because of its high oxidative profile [7] and its chronic contractile activity [38], the diaphragm generates great quantities of oxidants and may, therefore, be more vulnerable to oxidative stress [38], as suggested by a greater lipofuscin accumulation in the diaphragm compared to the vastus lateralis, both in controls and in patients with COPD. This increased lipofuscin count was found in both type I and type II of diaphragmatic fibres, likely because both types of fibres may have greater oxidative potential than corresponding fibres in the vastus lateralis [6]. Although oxidative muscles also have greater antioxidant capacities than less oxidative muscles [39], it is conceivable that the diaphragmatic antioxidant capacities were not sufficient to match its greater oxidant load. Recent studies performed in patients with COPD demonstrated the possibility that local exercises of the quadriceps induce oxidative stress within this muscle and that this could be associated with reduced muscular endurance [40]. To the extent that these findings could also apply to the diaphragm, chronic accumulation of oxidative stress in mildto-moderate COPD could enhance the possibility of diaphragmatic dysfunction later in the disease. Conversely, oxidative stress is not necessarily damaging for the muscle; in fact it may be necessary to trigger muscle adaptation [41]. The question of whether diaphragmatic oxidative stress is beneficial or detrimental for the diaphragm was beyond the scope of the present study and deserves further evaluation.

\section{Methodological considerations}

In human subjects, diaphragm samples needed to study its cellular adaptation to disease can only be obtained on patients scheduled to undergo surgical procedures. The presence of a lung neoplasm in the majority of participating subjects could, by itself or through cancer-related cachexia, influence muscle function. However, it is unlikely that the differences found between control subjects and patients with COPD could be explained on this basis, because cancer staging and the nutritional status, as assessed by BMI and albumin levels, were similar between the two groups. Some subjects were hospitalised before the surgery, raising the possibility that reduced levels of activity could have initiated a decrease in the vastus lateralis enzymatic activities, explaining, in part, the observed difference between the diaphragm and the vastus lateralis muscle. The most important justification for preoperative hospitalisation was to complete the medical assessment in patients living in areas remote from the hospital. These patients remained ambulatory while in hospital and benefited from general physiotherapy. It should also be noted that the differences in enzymatic activities found between the diaphragm and the vastus lateralis muscle still persisted when considering only those who were admitted to the hospital the day of the surgery in the analysis. Given the time-course of changes in fibre-type profile associated with reduced activity [42], it is unlikely that the observed changes in the fibre-type distribution observed between the diaphragm and the vastus lateralis muscle in COPD could be explained on the basis of a few days of preoperative hospitalisation.

\section{Conclusion}

The results of the current study suggest that muscle adaptation to chronic obstructive pulmonary disease occurs earlier in the diaphragm than in the vastus lateralis. This finding supports the notion that in mild-to-moderate chronic obstructive pulmonary disease, local factors, such as the level of muscle activation, may be more relevant to muscle adaptation than systemic factors, such as inflammation.

\begin{abstract}
Acknowledgements. The authors would like to acknowledge the contribution of A. Michaud for her technical assistance, S. Simard for his statistical assistance, and M. Bélanger, M-J. Breton, B. Jean, F. Simard and F. Faucher for their help in accomplishing this study.
\end{abstract}

\section{References}

1. Gosker HR, Wouters EFM, van der Vusse GJ, Schols AMWJ. Skeletal muscle dysfunction in chronic obstructive pulmonary disease and chronic heart failure: underlying mechanisms and therapy perspectives. Am J Clin Nutr 2000; 71: 1033-1047.

2. Whittom F, Jobin J, Simard PM, et al. Histochemical and morphological characteristics of the vastus lateralis muscle in patients with chronic obstructive pulmonary disease. Med Sci Sports Exerc 1998; 30: 1467-1474.

3. Maltais F, LeBlanc P, Jobin J, Casaburi R. Dysfonction musculaire périphérique dans la bronchopneumopathie chronique obstructive. Rev Mal Respir 2002; 19: 444453.

4. Orozco-Levi M. Structure and function of the respiratory muscles in patients with COPD: impairment or adaptation? Eur Respir $J$ 2003; 22: 41s-51s.

5. Levine S, Kaiser L, Leferovich J, Tikunov B. Cellular adaptations in the diaphragm in chronic obstructive pulmonary disease. N Engl J Med 1997; 337: 1799-1806.

6. Levine S, Gregory C, Nguyen T, et al. Bioenergetic adaptation of individual human diaphragmatic myofibers to severe COPD. J Appl Physiol 2002; 92: 1205-1213.

7. Ribera F, N'Guessan B, Zoll J, et al. Mitochondrial electron transport chain function is enhanced in inspiratory muscles of COPD patients. Am J Respir Crit Care Med 2003; 167: 873-879.

8. Simoneau JA, Bouchard C. Human variation in skeletal muscle fiber-type proportion and enzyme activities. $A m J$ Physiol 1989; 257: E567-E572.

9. Engelen MPKJ, Orozco-Levi M, Deutz NE, et al. Glutathione and glutamate levels in the diaphragm of patients with chronic obstructive pulmonary disease. Eur Respir $J$ 2004; 23: 545-551.

10. American Thoracic Society. Standards for the diagnosis and care of patients with chronic obstructive pulmonary disease. Am J Respir Crit Care Med 1995; 152: S77-S120.

11. Knudson RJ, Slatin RC, Lebowitz MD, Burrows B. The maximal expiratory flow-volume curve. Normal standards, variability and effects of age. Am Rev Respir Dis 1976; 113: 587-600.

12. Goldman HI, Becklake MR. Respiratory function tests: normal values at median altitudes and the prediction of normal results. Am Rev Tuberc 1959; 79: 454 467.

13. Cotes JE, Hall AM. The transfer factors for the lung; normal values in adults. In: Arcangeli $\mathrm{P}$, ed. Normal Values for Respiratory Function in Man. Torino, Panminerva Medica, 1970; pp. 327-343. 
14. Heymsfield SB, Williams PJ. Nutritional assessment by clinical and biochemical methods. In: Shils ME, Olson JA, eds. Modern Nutrition in Health and Disease. Philadelphia, Lea \& Febiger, 1994; pp. 812-841.

15. Bergström J. Muscle electrolytes in man: determination by neutron activation analysis on needle biopsy specimens. A study on normal subjects, kidney patients and patients with chronic diarrhoea. Scand J Clin 1962; 14: 1-110.

16. Maltais F, Simard AA, Simard C, Jobin J, Desgagnés P, LeBlanc P. Oxidative capacity of the skeletal muscle and lactic acid kinetics during exercise in normal subjects and in patients with COPD. Am J Respir Crit Care Med 1996; 153: 288-293.

17. Allaire J, Maltais F, LeBlanc P, et al. Lipofuscin accumulation in the vastus lateralis muscle in patients with chronic obstructive pulmonary disease. Muscle Nerve 2002; 25: 383-389.

18. Mabuchi K, Sreter FA. Actomyosin ATPase. II. Fibre typing by histochemical ATPase reaction. Muscle Nerve 1980; 3: 233-239.

19. Blomstrand E, Celsing F, Fridén J, Ekblom B. How to calculate human muscle fibre areas in biopsy samples: methodological considerations. Acta Physiol Scand 1984; 122: 545-551.

20. Havenith MG, Visser R, Schrijvers-van Schendel JM, Bosman FT. Muscle fiber typing in routinely processed skeletal muscle with monoclonal antibodies. Histochemistry 1990; 93: 497-499.

21. Green HJ, Sutton JR, Cymerman A, Young PM, Houston CS. Operation Everest II: adaptations in human skeletal muscle. J Appl Physiol 1989; 66: 2454-2461.

22. O'Donnell DE, Aaron S, Bourbeau J, et al. Canadian Thoracic Society recommendations for management of chronic obstructive pulmonary disease - 2003. Can Respir J 2003; 10: 37A-65A.

23. Mercadier JJ, Schwartz K, Schiaffino S, et al. Myosin heavy chain gene expression changes in the diaphragm of patients with chronic lung hyperinflation. Am J Physiol Lung (Cell Mol Physiol) 1998; 274: L527-L534.

24. Maltais F, LeBlanc P, Whittom F, et al. Oxidative enzyme activities of the vastus lateralis muscle and the functional status in patients with COPD. Thorax 2000; 55: 848-853.

25. Boczkowski J, Lanone S, Aubier M. Particularités de la cellule musculaire diaphragmatique. Rev Mal Respir 2000; 17: 569-573.

26. Mizuno M. Human respiratory muscles: fibre morphology and capillary supply. Eur Respir J 1991; 4: 587-601.

27. Sánchez J, Derenne JP, Debesse B, Riquet M, Monod H. Typology of the respiratory muscles in normal men and in patients with moderate chronic respiratory diseases. Bull Eur Physiopathol Respir 1982; 18: 901-1014.
28. Gayan-Ramirez G, Decramer M. Pertinence clinique de la répartition des isoformes de la myosine dans le muscle diaphragmatique. Rev Mal Respir 2000; 17: 574-584.

29. Poole DC, Sexton W, Frakas GA, Powers SK, Reid MB. Diaphragm structure and function in health and disease. Med Sci Sports Exerc 1997; 29: 738-754.

30. Orozco-Levi M, Gea J, Aguar MC, Broquetas JM. Changes in the capillary content in the diaphragm of COPD patients: a sort of muscle remodelling? Am J Respir Crit Care Med 1996; 153: A298.

31. Sánchez J, Medrano G, Debesse B, Riquet M, Derenne JP. Muscle fibre types in costal and crural diaphragm in normal men and in patients with moderate chronic respiratory disease. Bull Eur Physiopathol Respir 1985; 21: 351-356.

32. Widrick JJ, Trappe SW, Blaser CA, Costill DL, Fitts RH. Isometric force and maximal shortening velocity of single muscle fibers from elite master runners. Am J Physiol Cell Physiol 1996; 271: C666-C675.

33. Sohal RS, Wolfe LS. Lipofuscin: characteristics and significance. Prog Brain Res 1986; 70: 171-183.

34. Terman A, Brunk UT. Lipofuscin: mechanisms of formation and increase with age. APMIS 1998; 106: 265-276.

35. Sohal RS, Marzabadi MR, Galaris D, Brunk UT. Effect of ambient oxygen concentration on lipofuscin accumulation in cultured rat heart myocytes - a novel in vitro model of lipofuscinogenesis. Free Radic Biol Med 1989; 6: 23-30.

36. Blackett AD, Hall DA. Tissue vitamin E levels and lipofuscin accumulation with age in the mouse. J Gerontol 1981; 36: $529-533$.

37. Gao G, Öllinger K, Brunk UT. Influence of intracellular glutathione concentration on lipofuscin accumulation in cultured neonatal rat cardiac myocytes. Free Radic Biol Med 1994; 16: 187-194.

38. Heunks LMA, Dekhuijzen PNR. Respiratory muscle function and free radicals: from cell to COPD. Thorax 2000; 55: 704-716.

39. Powers SK, Leuwenburgh C. Exercise training-induced alterations in skeletal muscle antioxidant capacity: a brief review. Med Sci Sports Exerc 1999; 31: 987-997.

40. Couillard A, Maltais F, Saey D, et al. Exercise-induced quadriceps oxidative stress and peripheral muscle dysfunction in patients with chronic obstructive pulmonary disease. Am J Respir Crit Care Med 2003; 167: 1664-1669.

41. Reid MB. Plasticity in skeletal, cardiac, and smooth muscle invited review: redox modulation of skeletal muscle contraction: what we know and what we don't. J Appl Physiol 2001; 90: 724-731.

42. Berg HE, Larsson L, Tesch PA. Lower limb skeletal muscle function after 6 wk of bed rest. J Appl Physiol 1997; 82: 182188. 